\title{
TÉCNICAS DE PRONÓSTICO Y ESTUDIOS DE MODERNIZACIÓN
}

José Manuel Merino E.*

\section{INTRODUCCIÓN}

Este trabajo está orientado a la descripción de las principales técnicas de pronóstico sociocultural y a sus vínculos potenciales con investigaciones atingentes al campo de la Modernización.

Los propósitos consensuales de la ciencia incluyen la descripción, explicación y predicción de fenómenos. En ese contexto, la indagación acerca de la naturaleza, características y principales enfoques técnicos del pronóstico, constituye un aspecto legítimo, deseable y necesario, en la actividad del científico, particularmente entre aquellos que de alguna manera están identificados con la transformación de rasgos individuales, institucionales, organizacionales y sociales. En el estudio científico de la Modernización adquiere así especial interés el conjunto de instrumentos y teorías que permiten el diseño anticipatorio de estrategias de cambio social y que fundan la planificación prospectiva sobre bases relativamente serias y adecuadas a las tendencias reales que se pueden constatar en las distintas dimensiones del acaecer social.

Esta actividad prospectiva no es algo novedoso o relativamente reciente en el ámbito de las Ciencias Sociales. En efecto, ha sido tradicional considerar el pensamiento utópico como la antesala verdadera de la prognosis científica. Los escritos de Platón en La República, de Tomás Moro, en Utopia de Campanella, en La Ciudad $\mathrm{de} / \mathrm{So} /$ de Foustel de Coulanges, etc., presentan asomos de estudios futuristas con visos distintos de certidumbre lógica, con rasgos diferentes de acercamiento a la

* El autor, Dr. en Sociología, pertenece a la U. de Concepción. 
futurología científica que hoy nos ocupa. En todo caso, estos precursores nos indican que la prospectiva ha sido una actividad siempre presente en la dinámica humana y que expresa fielmente la curiosidad del hombre por el conocimiento y manipulación del futuro, especialmente en sus dimensiones societales.

Sin embargo, en ninguno de estos precursores se ha encontrado, salvo por azar, la utilización de instrumentos rigurosos, de consistencia metodológica probada y, por tanto, de valor indiscutible en la exploración del futuro. Esas características, no totalmente presentes en los intentos contemporáneos predictivos, constituyen no obstante los requisitos básicos hacia los cuales se orientan los prognósticos más reconocidos. Las exigencias metódicas y técnicas que caracterizan los enfoques futuristas de hoy son tal vez la prueba más irrecusable de su validez como herramienta en la planificación del cambio social en todas sus esferas, y niveles.

\section{LAS TÉCNICAS DE PRONÓSTICOS}

Desde la década de 1960, los estudios de prospectiva se han extendido enormemente, abarcando innumerables tópicos y dando lugar a considerables refinamientos técnicos. Desde las simples extrapolaciones y afirmaciones de tendencias hasta los más sofisticados métodos cuantitativos existe un amplio campo, que procuraremos revisar en la perspectiva de explorar los principales enfoques prognósticos $y$ sus posibles aplicaciones en el terreno de la Modernización.

Sin pretender ser exhaustivo en esta revisión de técnicas de pronóstico, concuerda con Chambers ${ }^{1}$ la existencia de tres tipos básicos de técnicas de pronóstico: técnicas cualitativas, análisis y proyección de series temporales y, por último, los modelos causales.

Las Técnicas Cualitativas de Pronóstico utilizan datos de esa naturaleza (opinión de expertos, por ejemplo), recolectan información acerca de asuntos específicos y pueden o no tomar en cuenta el pasado en su proyección del futuro.

El segundo conjunto de procedimientos -el Análisis y Proyección de Series Temporales - se focaliza por completo en el examen de patrones de cambio y depende en forma decisiva sobre datos históricos.

El tercer enfoque prospectivo utiliza información específica, altamente refinada, acerca de relaciones entre elementos de un sistema y, aunque es el más poderoso, requiere tomar resguardos formales. Como la técnica anterior, el pasado es un elemento importante en los modelos causales.

Entre los Métodos Cualitativos de Pronóstico se pueden enunciar los siguientes: el Método Delphi, la Investigación de Mercado, el Panel, el Pronóstico Profético o Visionario y la Analogía Histórica. Entre los métodos de Análisis y Proyección de

1 Chambers, J.C. y Mullick, S.: How to choose the right forecasting technique. Harvard Business Review, July. August 1971, pp. 45-74. 
Series Temporales, podemos enumerar: el Método de Promedios Móviles, las Curvas Exponenciales, el Método Box-Jenkis, las Proyecciones de Tendencias y el Método X-11. Los principales Métodos Causales incluyen el Modelo de Regresión, el Modelo Econométrico, el Modelo Input-Output, el Índice de Difusión y el Análisis de Ciclo Vital.

Evidentemente, estos distintos procedimientos y recursos de pronósticos tienen distintos niveles de exactitud, de aplicaciones, de necesidades de información, de costo, de tiempo requerido para el desarrollo de los pronósticos, etc. Asimismo, han sido aplicados en distintos dominios sociales con diferentes resultados finales. Lo importante es constatar el amplio espectro de recursos técnicos que en modo alguno agota todas las posibilidades conocidas.

Es importante también destacar la institucionalización creciente de los estudios prospectivos que ha sido correlativa al desarrollo de estas técnicas. Existen actualmente y desde hace algunas décadas importantes Centros de Investigación Futurológicos que han creado verdaderas escuelas en el tratamiento técnico de los pronósticos: Ia RAND CORPORATION y el hUDSON INSTITUTE en EE.UU., el Centro de Estudios de Prospectiva en Francia, el Departamento de Futurología de la OCDE en Europa, son algunos de los más destacados ejemplos de estas organizaciones dedicadas por completo al estudio del futuro.

Con el objeto de precisar con algún detalle las técnicas de pronóstico que, a mi juicio, son más relevantes procuraré describir los rasgos sobresalientes de estos procedimientos principales. En particular, es de interés establecer algunos comentarios acerca de los procedimentos prospectivos que tienen más inmediata aplicación en Ciencias Sociales; examinaremos la Técnica Delphi, el método Box-Jenkis y el Análisis de Guiones, que son a grandes rasgos, los recursos pronósticos más vinculados en la actualidad a los estudiosos de nuestras disciplinas.

\section{MÉTODO DELPHI}

El Método Delphi es un procedimiento sistemático para obtener opiniones de expertos acerca de algunos temas particulares. Fue diseñado en la RAND CORPORATION ${ }^{2}$, en la década de 1950, para estudiar algunos problemas estratégicos, por razones de seguridad nacional sólo ha sido difundido internacionalmente a partir de 1962. Desde entonces, ha despertado mucho interés en las Universidades, gobiernos e industrias por su capacidad predictiva en vastos asuntos de la vida sociocultural y tecnológica.

2 Dalkey, Norman: Delphin. Rand Corp. Santa Mónica, 1967, p. 3904; The Delphi Method, ibíd., p. 5888; Experiments in group prediction, ibíd., p. 3828. También: Dalkey y Helmer: An experimental application of the Delphi Method to the use of experts. Management Since vol. 9, 1963, pp. 458-467. 
Su nombre se deriva del antiguo oráculo griego y ha sido empleado para obtener opiniones sobre lo que nos depara el futuro; pero su alcance es más general ya que puede ser usado en cualquier contexto donde se busca un consenso de opiniones entre expertos sobre una materia específica.

En términos generales, el Método Delphi tiene tres características esenciales: 1) Anonimato; 2) Retroalimentación controlada y 3) Respuesta Estadística de Grupo.

Consiste en la selección de un conjunto de expertos que localiza el interés de la investigación; en la aplicación de una serie de cuestionarios a dichos expertos, en vueltas sucesivas, comunicándole a cada experto en forma previa los resultados de la vuelta anterior. Evidentemente, las personas involucradas en el estudio - los expertos-desconocen al resto de los participantes y sólo conocen los resultados globales de sus respuestas en cada vuelta. Cada experto en virtud del conocimiento que recibe de la respuesta del grupo puede mantener, modificar o fundamentar la propia, refinando de ese modo cada vez más el consenso del grupo respecto de la materia investigada.

Las características principales del Método Delphi permitan, al ser examinadas individualmente, algún grado de interiorización mayor acerca de la naturaleza de sus procedimientos. Observemos, por tanto, estas características:

\section{Anonimato}

El procedimiento típico consiste en aplicar cuestionarios a los expertos, en forma individual. Este recurso implica que los expertos no son puestos en contacto personal y en situaciones de debate cara a cara, sino que participan en el estudio de modo estrictamente separado. Se elimina de este modo el conjunto de sesgos provenientes de la influencia ejercida en un grupo por individuos dominantes; se controlan además de modo directo los efectos de grupo sobre el comportamiento individual: la tendencia a defender a muerte las ideas personales, la propensión a ser persuadido por individuos elocuentes, etc.

\section{Retroalimentación controlada}

La retroalimentación se logra en virtud de la ejecución de varias vueltas del ejercicio de encuestas: Las opiniones generales durante cada una de ellas son retroalimentadas al grupo en la vuelta siguiente mediante la entrega a los participantes de un resumen de los resultados de la vuelta previa, generalmente en forma de resúmenes estadísticos. Este hecho de retroalimentar información refinada a los expertos tiene varias ventajas teóricas que han sido probadas en decenas de experimentos:

a) En casi todos los casos existe una marcada convergencia entre las opiniones y la retroalimentación. En la ronda inicial, las opiniones tienden a difundirse en 
un amplio espectro. Esta difusión tiende a disminuir en forma evidente en las vueltas siguientes.

b) La disminución principal ocurre entre la primera y segunda vuelta.

c) Lo más significativo es que la exactitud de las respuestas de grupo aumenta con la reiteración.

\section{Respuesta estadística de grupo}

La opinión del grupo se da a conocer con una expresión estadística, usualmente la Mediana y el Rango Intercuartílico; el empleo de una definición estadística de la respuesta de grupo es una forma de disminuir la presión que conduce a la conformidad. La utilización de la Mediana indica al experto el valor promedio del grupo en la situación examinada, el Rango Intercuartílico le indica la dispersión existente bajo y sobre la Mediana. Este hecho hace que el sujeto pueda estimar su posición inicial y refinar su juicio de un modo analítico en las vueltas posteriores.

Al finalizar el ejercicio, aún puede haber una importante dispersión de las opiniones individuales, lo que probablemente reviste mayor significación es que la respuesta estadística de grupo es una técnica para asegurar que la opinión de cada miembro esté representada en la respuesta final. No existe presión alguna para llegar al consenso.

Existen diversas propiedades del método Delphi que deberían ser destacadas. Ante todo, el procedimiento es una forma rápida y relativamente eficaz de extraer el "summum" de un grupo de eruditos. En términos generales, para el participante, responder a un cuestionario bien diseñado implica mucho menos esfuerzo que participar, por ejemplo, en una conferencia o escribir un artículo; un ejercicio Delphi, manejado en forma apropiada, puede ser altamente motivador para los encuestados. La retroalimentación puede resultar novedosa e interesante para todos, si existe respeto mutuo en el grupo de expertos comprometidos. El uso de procedimientos sistemáticos hace que los resultados - que pueden o ser genuinos o no- aparezcan como objetivos. Aún así, este uso significa, por lo menos, una garantía.

Por último, el anonimato y la respuesta de grupo permiten compartir responsabilidades, cosa que resulta estimulante y libera a los encuestados de sus tensiones. Se puede afirmar con certeza que los resultados de la aplicación del método Delphi están sujetos a mayor aceptación por parte del grupo que los acuerdos a los que el mismo grupo llegue mediante formas de interacción más directas.

En los últimos años, se ha observado un creciente uso del método Delphi en distintas esferas de investigación: el pronóstico sociocultural, la opción tecnológica, el pronóstico en asuntos de salud, medio ambiente, comercio, etc. Tal vez su área de mayor desarrollo continuo en los últimos años ha sido en los dominios tecnológicos, sin embargo, en el campo sociocultural tiene amplias ventajas que 
serán desarrolladas en los próximos tiempos. En general, en las Ciencias Sociales tan desprovistas de instrumentos técnicos poderosos para explicar y predecir los fenómenos de su materia-objeto, los métodos Delphi pueden significar un recurso valioso en la predicción de fenómenos sociales y culturales del mayor interés ${ }^{3}$.

\section{TÉCNICA BOX-JENKINS}

El procedimiento de pronóstico establecido por Box y Jenkins ${ }^{4}$ está asociado al análisis y proyección de Series Temporales. Se vincula muy estrechamente al análisis secundario utilizado en Sociología y permite un tratamiento formalizado de los Indicadores Sociales, temática de gran desarrollo actual en Sociología y Planificación Social.

La técnica específica ha sido denominada ARIMA (Autoregressive Process to Moving Average) y consiste en el ajuste de una función matemática a una serie estadística inicial que combina un proceso de autorregresión con una determinación de promedios móviles; este ajuste funcional permite desarrollar un conjunto de fórmulas algebraicas que perfilan en términos muy exactos los valores pronosticados para años futuros en la serie estadística considerada.

La serie temporal es ajustada en este caso con un modelo matemático que es óptimo en el sentido que permite reducir al mínimo los errores tendenciales, más que en cualquier otro modelo. El tipo de modelo debe ser identificado y entonces deben ser estimados sus parámetros. Aparentemente ésta es la técnica estadística más exacta de que se dispone actualmente, aunque frente a otros procesos de pronóstico es mucho más cara y significa un proceso de análisis considerablemente superior en el terreno temporal.

La exactitud de esta forma de pronóstico ha sido valorada entre muy buena y excelente. Sus aplicaciones principales han estado centradas en la predicción de tendencias estadísticas y de indicadores sociales tan importantes como antecedentes demográficos, migratorios, tasa de suicidios, de delincuencia, de densidades de población, de concentración urbana, etc. Entre los estudios más destacados, efectuados en el Campo de las Ciencias Sociales, mediante el método Box-Jenkins se destaca el efectuado por Vigderhous en 1978 acerca de los movimientos futuros de la tasa de suicidios en EE.UU.

3 Ver: Helmer, Olaf. Analysis of the future: the Delphi Method. Rand Corp. Santa Mónica, 1967, p. 3558 y Opinión médica sobre el aborto en Jamaica, un encuentro Delphin. Estudios de Poss vol. 1, No 12, Colombia, 1970.

4 Box, G.E.P. y Jenkins, G.M.. Time series analysis o Forecarting and control. Halden Day, San Francisco, 1976. 


\section{EXTRAPOLACIONES Y GUIONES TENDENCIALES}

En el dominio de las Ciencias Sociales, se ha destacado con brillo propio desde hace varias décadas el trabajo prospectivo realizado por Herman Kahn del Hudson Institute ${ }^{5}$.

El método desarrollado por Kahn ha sido fundamentalmente la extrapolación de tendencias y el diseño de guiones apropiados para predecir el acaecer de esas tendencias. Entre los variados modelos de pronóstico social éste constituye uno de los más simples por su naturaleza eminentemente analítica y su exclusión de sofisticados modelos estadísticos. No debemos olvidar que Kahn es historiador y que este punto de vista se emplea con mucha intensidad en sus estudios y pronósticos.

Sin embargo, la eficacia de las proyecciones históricas del Hudson Institute están más que probadas mediante el hecho pronosticado hace varias décadas por Kahn acerca de Japón en el liderato económico mundiat.

En términos técnicos, la prospectiva del Hudson Institute se caracteriza por considerar que la historia constituye una especie de almacén de materias primas para la prospectiva y que determinados periodos son particularmente ejemplares para el porvenir. Así, la civilización grecorromana ofrece un ejemplo de solución a las contradicciones socioculturales; la Roma de Augusto ejemplifica una renovación ideológica sobre las cenizas de un sistema político moribundo; $y$, finalmente, la Belle Époque -que los autores sitúan entre 1900 y 1913 - ilustra lo que ocurre cuando el mundo está libre de guerras y el crecimiento es relativamente rápido. Este último ejemplo es el elegido por los investigadores del Hudson para suponer lo que podría ser la civilización postindustrial, imaginándola como una "tercera" Belle Époque. Esta "tercera" Belle Époque, no aparecería, según dichos investigadores, más que con un nivel de vida postindustrial equivalente a una renta media situada entre diez mil y veinticinco mil dólares (1969), situación que en el año 2000 podría darse en Japón, Europa Occidental (excluidas Gran Bretaña, Luxemburgo y Austria), la Alemania del Este, Estados Unidos y Canadá.

Mientras llegue el momento, los autores prevén para los años ochenta acontecimientos como éstos: afirmación del Japón como superpotencia, mantenimiento de una rápida progresión del Producto Nacional Bruto en las regiones "dinámicas" e industrializadas, riesgos de una confrontación chino-japonesa a causa de la creciente importancia de una zona capitalista de dominación nipona en Asia, Francia como líder de Europa, etc.

\section{CONCLUSIONES}

En este recuento y análisis de la Prospectiva se ha intentado realizar un examen de

5 Kahn, Herman. Hacia el año 2018. Emecé, B. Aires, 1969. 
los principales recursos disponibles para el pronóstico científico en el ámbito sociocultural. No se ha estimado que sea conveniente incluir estudios y enfoques futurológicos sin una sólida base técnica que la diferencie sitemáticamente de la simple profecía o de la especulación imaginativa. En ese sentido, se puede estar de acuerdo con Schuessler ${ }^{6}$ en que aún falta mucho tiempo para que las Ciencias Sociales dispongan de instrumentos prospectivos de validez y confiabilidad reconocida; sin embargo, creemos que existen algunos desarrollos técnicos que abren un sendero y precisamente son las que se ha tratado de aclarar en sus dimensiones centrales.

No se escapa al hecho que los estudios prospectivos han tenido ya un considerable impacto en ciertas esferas sociales de los países desarrollados. Conocemos la trascendencia del Informe al Club de Roma con el estudio acerca de los límites del crecimiento preparado hace algunos años por Meadows en el Massachusset Institute of Technology (M.I.T.) ${ }^{7}$ que introdujo en el mundo la preocupación por los recursos no renovables que comienzan a extinguirse y creó una conciencia ecológica creciente que condujo a la formulación de la noción de Eco desarrollo y Calidad de Vida, entre otros. Tampoco extraña la idea del considerable debate, asociado a lo anterior, que se ha generado en términos de la Sociedad Postindustrial con enfoques pesimistas y optimistas, entre los cuales los trabajos de Heilbroner, Toffler y otros han adquirido gran notoriedad.

Sin embargo, la urgencia actual en el terreno de las Ciencias Sociales y particularmente en el campo vinculado a la Modernización, pone en la lista de prioridades la consideración de los procedimientos y recursos instrumentales que son opcionales al estudio de pronósticos. Schuessler, Keul. Quantitative Methodology in Sociology. American Behavioral Scientist. July, August, vol. 23, No 6, pp. 835-860, 1980.

7 Meadows, Dennis. Los límites del crecimiento. FCE, México, 1970. 